\title{
Phylogenetic Analysis of Spike and Envelope Proteins for a Number of Bat Coronaviruses for Understanding the Hypothesis of Possible Origin for the Novel 2019- $\mathrm{nCoV}$
}

\author{
Salar Ibrahim Ali \\ Nursing Department \\ Technical College of Health \\ Sulaimani Polytechnic University \\ Sulaimani, Iraq \\ salar.ali@spu.edu.iq
}

\begin{tabular}{l} 
Article Info \\
\hline Volume 5-Special Issue: $4^{\text {th }}$ \\
International Conference on the Health \\
and Medical Science : Medical \\
Researches Improve Life Quality \\
(ICHMS 2020) \\
DOI: \\
10.24017/science.2020.ICHMS2020.15 \\
Article history: \\
Received: 08 September 2020 \\
Accepted: 22 September 2020
\end{tabular}

Keywords:

COVID-19, Rousettus bat, Bat coronaviruses, Phylogenetic tree analysis, Amino acid alignments.

\begin{abstract}
Coronavirus Disease 19 (COVID-19) emergence reveals globally a great health issue and due to the limited information and knowledge on the origin of this novel coronavirus 2019 (2019-nCoV). Therefore, this study aims to investigate the evolution and analysis of molecular epidemiology for both Spike and Envelope proteins of 20 available complete genome sequences of different bat coronaviruses including 2019-nCoV in order to find out which type of bat coronaviruses is more likely to be the origin of this new 2019-nCoV and also multiple amino acid sequences of Envelope protein for all bat coronaviruses were aligned for the purpose of finding the greater probability of novel 2019-nCoV original host among bat coronaviruses. Phylogenetic tree analysis for Spike protein revealed that all 2019$n C o V$ related coronaviruses isolated from these species of species are discovered in China and Hong Kong and the Middle East bat are less likely to contribute in spreading or to become the origin of 2019-nCoV and all coronaviruses that from Hong Kong and China are located into one clade next to the clade that contains 2019-nCoV coronaviruses which indicates that this group of coronaviruses are genetically different for 2019-nCoV; moreover, Hong Kong and USA bat coronaviruses does not contain the bat coronavirus from China and are located into
\end{abstract}


one clade far from the clade that contains 2019-nCoV indicates that all coronaviruses are genetically very different from 2019-nCoV, and USA bat coronavirus may has no role in generating of 2019-nCoV. The phylogenetic trees analysis of Envelope protein showed that Envelope protein of different coronaviruses are more similar in comparison to Spike protein, USA bat coronavirus has a relatively closeness relationship to 2019-nCoV. Furthermore, Envelope protein alignment showed the closely related amino acid sequence which confirms that the outcomes of phylogenetic tree analysis in which that these bat coronaviruses have genetically close relationship together and more interestingly amino acid sequence (MG772934.1) shows 100\% identity with the amino acid sequence of 2019-nCoV (NC 045512.2) and the same virus has a close relationship in both Spike and Envelope due to that in both phylogenetic tree analysis are neighbored with 2019 nCoV in the same clade.

Copyright (C) 2020 Kurdistan Journal of Applied Research. All rights reserved.

\section{INTRODUCTION}

During late period in December 2019, the Chinse authority was announced epidemic outbarking of the novel severe acute respiratory syndrome like pneumonia, later on the name of Coronavirus Infectious Disease 19 (COVID-19) is known by World Health Organization (WHO) is named as Coronavirus Infection Disease 19 (COVID-19) that revealed a global alert as the most transmissible and infectious disease. The Severe Acute Respiratory Syndrome-Coronavirus-2 (SARS-CoV2) was considered as zoonotic diseases which is thought to be transmitted from Bat and Pangolin (intermediate host). Subsequently, it was firstly observed that the wet seafood in the Wuhan city market of China caused the infection to human and also the transmission of the infection between humans was confirmed in early days during its spread [1,2]. The discovery of the novel SARS-CoV in 2003 was identifed as a causative agent for the outbreak of SARS [1-3]. In Wuhan city of China, there has been a record of cases of severe acute lung inflammation, and this infection was caused because of the new coronavirus (2019-nCoV) emergence and responsible for causing acute human respiratory infections. A number of different pneumonia cases of undefined origin related to permanence have been reported in the Wuhan seafood markets since the beginning of this viral infection [2-4]. The 2019-nCoV belongs to subfamily of Orthocoronavirinae, which has the distinctness of MERS-CoV and SARS-CoV [4,5]. Until present, the global confirmation of the total of $19,936,210$ respiratory infection cases has been reported, and mortality is 732,499 [6,7]. The pandemic outbreak of $2019-\mathrm{nCoV}$ is contributed by the zoonotic transmission; for instance, a high number of infected people in China stated a visiting in November to Wuhan seafood markets. In the recent time, research evidence has been reported that this new coronavirus can be transmitted from animals to human and transmission among humans themselves [7,8]. Furthermore, most transmission of SARS between medical intensive care staff was in fact related with the respiratory air droplets of suffered people from the infection [9]. Moreover, in China the COVID-19 cases raise and high death rate are likely because of health care system failing, that involves the enhancement and education of public to practice protection interventions, including keeping social distance and restrictions of movement [10]. In an epidemiological investigation, it has been revealed that this $2019-\mathrm{nCoV}$ has the ability in causing the infection in age groups and in a variety of different geographical places [11] and it has been showed that the transmission of disease can be directly and indirectly through contaminated materials [12]. As it is observed in COVID-19 patients, there are a different response of the patient to the infection that ranges from lack of symptoms to severs lung infection and such as conditions were observed too in the Middle East with MERS outbreaks $[12,13]$. 
Prompt techniques of identification and quarantine and patients have taken a consideration as the distinctive feature during the preliminary phases of infection control and management of the novel viral pandemic outbreak. Moreover, the estimation of genetic variability for the novel coronavirus 2019-nCoV and its rate of evolution, and phylogenetic analysis poses the important findings for understanding the progression of infection, discovery of antiviral treatment, and vaccine development. this study aims to perform the phylogenetic trees analysis of both Spike and Envelope protein for a number of selected bat coronaviruses including 2019-nCoV created by using the 20 available complete genome sequences of bat coronaviruses including the $2019-\mathrm{nCoV}$ in order to find out which type of bat coronaviruses have the relative closeness relationship with the $2019-\mathrm{nCoV}$ and could has the possibility to be the origin the 2019-nCoV and finally Envelope protein amino acid alignment were performed for the selected bat coronaviruses in order to understand and find out the possible real origin of this new human coronavirus (2019-nCoV).

\section{METHODS AND MATERIALS}

20 available complete genome sequences for a number of bat coronaviruses and the novel human coronavirus (2019-nCoV) downloaded from GenBank (http://www.ncbi.nlm.nih.gov/genbank/), (EF203067.1 Bat/HKU2/CHN/2006, EF203065.1 Bat/ CHN/2006, EF203064.1 Bat-HKU2/GD/CHN/2006, NC 009988.1 Bat/HKU2/CHN/2007, MF370205.1 Rhinolophus bat/HKU2/Guangdong/CHN/2017, JQ989271.1 Rousettus bat/HKU10/CHN/2005, JQ989271.1 Rousettus bat/HKU10/CHN/2012, NC 030886.1 Rousettus bat/CHN/2016, NC 762337.1 Rousettus bat/CHN/2018, NC 030886.1 Rousettus bat/Beijing/CHN/2018, NC 009021.1 Bat/HKU91/CHN/2007, MG762674.1 Rousettus bat/HKU9/Jinghong/CHN/2009, NM211101.1 Bat/HKU-9-10-2/CHN/2010, NC 022102.1 Bat-CDPHE15/USA/2006, NC 045512.2 COVID19/Wuhan-Hu-1/CHN/2019, MG 772934.1 Bat SARS-like coronavirus isolate bat-SLCoVZXC21/CHN/2020, KY417146.1 Bat SARS-like coronavirus isolate Rs4231/CHN/2016, MK211376.1 Coronavirus BtRs-BetaCoV/YN2018B/CHN/2018， DQ648857.1 Bat coronavirus BtCoV/279/CHN/2005 and DQ022305.2 Bat SARS coronavirus HKU31/HK/CHN/2005). Similarity percentage identified using basic local alignment search tools (https://blast.ncbi.nlm.nih.gov/Blast.cgi). Duplicated and very similar sequences excluded from the datasets. Sample size is 20 sequences and aligned using multiple sequence alignment tool and manually edited using BioEdit program v7.0.5. [14]. The analyses of protein sequences was performed by Maximum likelihood (ML) methods due to its importance for estimating the hypotheses of different phylogenetic through calculation of the given model probability evolution that generated from the available information. Likelihood ratio test used for comparison of the nested models probabilities. The construction of ML tree was performed by generalized time-reversible plus gamma distribution and MEGA-X was used as evolutionary model for invariant sites $(+\mathrm{G}+\mathrm{I})$ [15]. Also, full amino acid sequence length for envelope of different selected bat coronaviruses including the 2019-nCoV aligned using multiple sequence alignment tool (MEGAX) and Clustal $W$ alignment [15].

\section{RESULTS}

For understanding different coronaviruses origin which including the 2019nCoV, 20 available entire sequences of amino acids of bat coronaviruses were studies via phylogenetical analysis and Clustal $W$ alignment. As it is clarified in (Figures 1 and 2); isolated coronaviruses from bat and all bat coronaviruses clade in separated cluster groups (Figure 1A, B and C), but all 2019-nCoV related coronaviruses are located in one group.

\section{Spike Protein}

All bat coronavirus sequences used in this study are obtained from different countries (Hong Kong, China, Egypt, and Africa) from different species of bat. All bat coronaviruses analysed are divided into three large groups (Figure 1A, B , C). For group A, the 2019-nCoV of human can be seen gathered with many other isolated coronaviruses from different bat species. As it is seen in cluster A (Figure $1 \mathrm{~A}$ ), all different bat-coronaviruses observed that cluster together and have very close relationship with 2019-nCoV. Different species of bat which are carrier for different coronavirus strains are Rousettus bat (Fruit bat), bat-HKU9-10 strain, bat-SLCoVZXC21, BtRs-BetaCoV/YN2018B, Rhinolophus bat. It is clear that all the 2019-nCoV related coronaviruses isolated from these species of bat are discovered in the mainland of 
China and Hong Kong. There are fruit bats (Rousettus bat) in Africa and Middle East but none of them are clustered in group A; Therefore, the Middle East bat are less likely to contribute in spreading or to become the origin of 2019-nCoV.

For Group B, it contains a smaller number of different bat coronaviruses. Coronaviruses; Batcoronavirus BtCoV/279, Bat-SARS coronavirus HKU3, and Bat SARS-like coronavirus isolate Rs4231 are claded with each other. All bat-coronaviruses of this group come from bats of Hong Kong and China isolated in different regions of China revealed in clade next to clade that contains 2019-nCoV coronaviruses. Therefore, this group collected all coronaviruses that genetically are different from 2019-nCoV.

Group $\mathrm{C}$ possess seven different bat-coronaviruses. In this group, coronavirus; Bat coronavirus come from bat of Hong Kong and USA but it does not contain the bats from China. Coronaviruses of this group are located into one clade far from the clade that contains 2019-nCoV coronaviruses. Therefore this group collected all coronaviruses that genetically are very different from $2019-\mathrm{nCoV}$ and USA coronavirus isolated from bat may not has a role in generating of the 2019-nCoV.

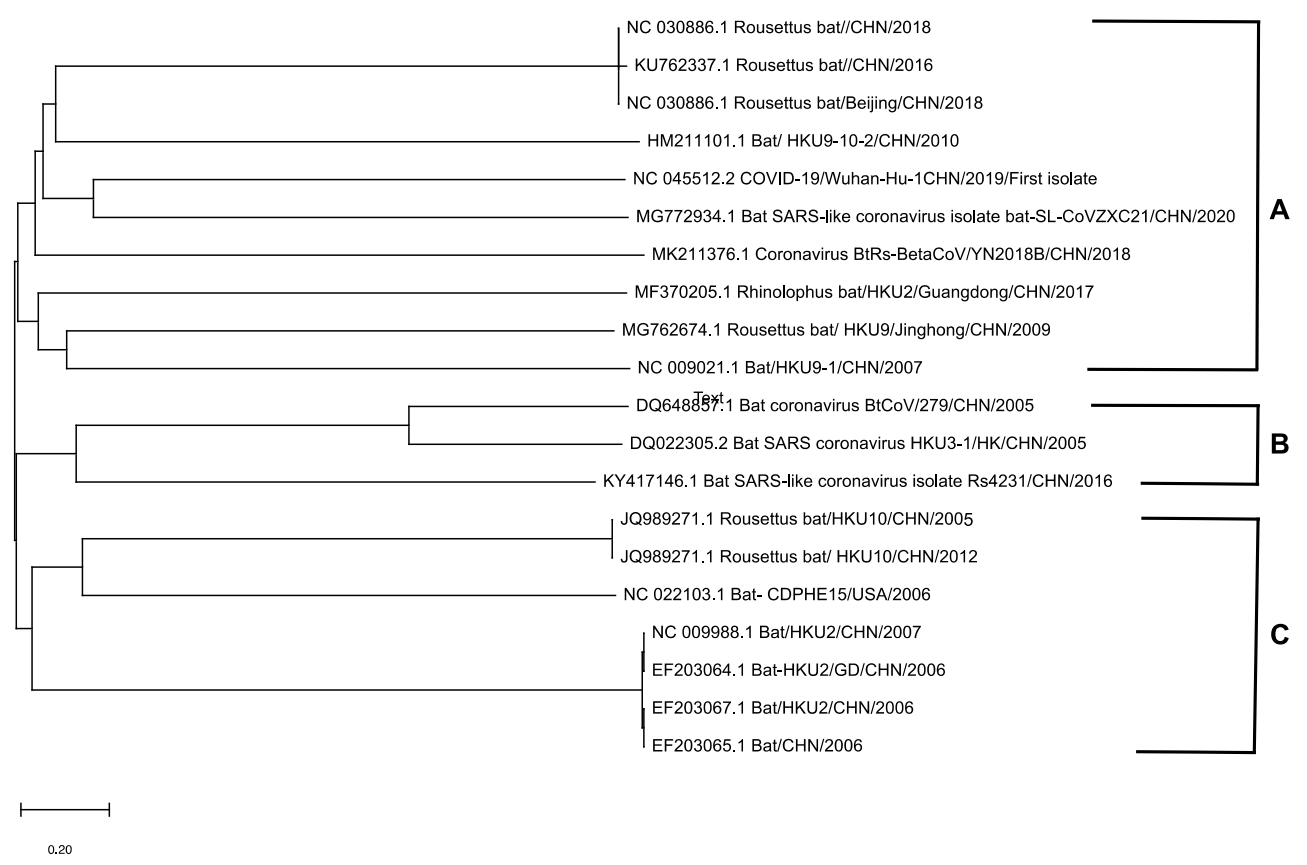

Figure 1: Phylogenetic tree analysis of Spike protein for a number of common bat-coronaviruses including 2019-nCoV. Phylogenetic tree was created by using Maximum Likelihood Method with PhyML and TreeDyn (www.phylogeny.fr). MUSCLE was applied for Protein domain sequences alignments

\section{Envelope Protein}

Envelope amino acid sequences of bat coronaviruses downloaded from NCBI which originated from different species of bat in different countries. The whole bat-coronaviruses including 2019-nCoV are located in two different groups of clusters (Figure 2 A, B). In group A, all bat coronaviruses that clustered in this group are genetically far from the group that gathered in the clade that containing the 2019-nCoV. Group B possess seven different bat- coronaviruses in China and USA. This group is composed of two sub clades. One clades contains more genetically relates coronaviruses but all are isolated from China and Hong Kong, and the second sub clade contains coronaviruses of bat that found in USA. It shows that the Envelope protein of different coronaviruses are more stable and more similar in comparison to Spike protein. In addition, USA coronavirus isolated from bat has relatively closeness related to the $2019-\mathrm{nCoV}$ but it is located in different sub clade and its spike sequence is very different and very far from to be concluded that USA bat coronavirus is related to the spreading of the 2019-nCoV. 


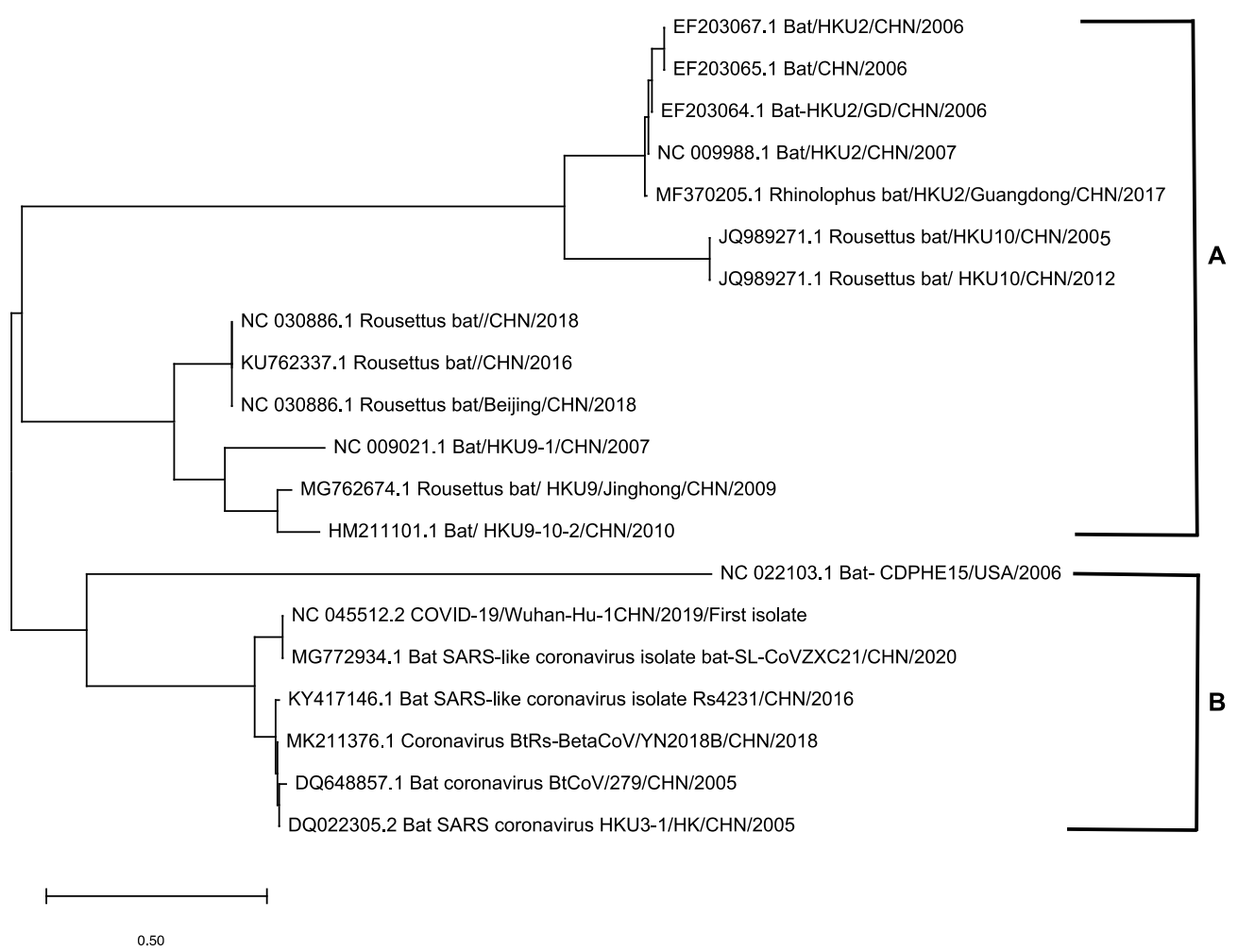

Figure 2: Phylogenetic tree analysis of Envelope protein for a number of common bat-coronaviruses including 2019-nCoV. Phylogenetic tree was created by using Maximum Likelihood Method with PhyML and TreeDyn (www.phylogeny.fr). MUSCLE was applied for Protein domain sequences alignments

\section{Multiple Amino Acid Sequence Alignments of Envelope Protein}

The analysis of the multiple sequence alignments of the full length for Envelope amino acid between different bat coronaviruses and including the 2019-nCoV. The amino acid alignments showed that all bat coronaviruses (NC 045512.2, MG772934.1, KY417146.1, MK211376.1, DQ648857.1, and DQ022305.2) that clustered in group B (Figure 2) have a similarity in amino acid sequence except that two amino acid have mutated. This closely related amino acid sequence confirms that the results of phylogenetic tree analysis in which that these bat coronaviruses have genetically a close relationship together. Interestingly, one of these amino acid sequences, (MG772934.1) is 100\% identical with the amino acid sequence of the 2019n$\mathrm{CoV}$ (NC 045512.2). The same virus has a close relationship in both Spike and Envelope amino acid sequence because in both phylogenetic tree analysis (Figure 1 and 2) are neighbored with the 2019n-CoV (NC 045512.2) in the same clade. Therefore, this bat coronavirus, Bat SARS-like-coronavirus isolate (bat-SL-CoVZXC21) has a greatest probability to be the origin of the $2019-\mathrm{nCoV}$ which are found in China but this type of bat is not exist in Iraq. 


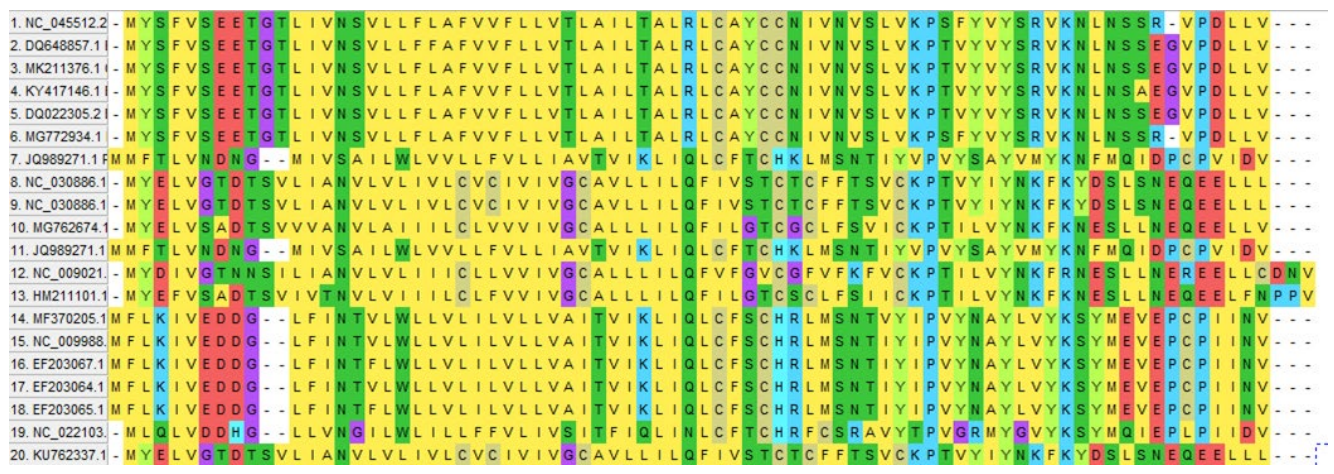

Figure 3: Multiple amino acid sequence alignments of Envelope for different selected types of batcoronaviruses involving 2019-nCoV and these amino acid sequence alignments is created by Clustal $\mathrm{W}$ alignment.

\section{DISCUSSION}

Due to COVID-19 pandemic outbreak, it has crucially importance in understanding the epidemiological characteristics of the $2019 \mathrm{nCoV}$. Therefore, this current investigation studies and aims to find out the more likely possible origin of the new $2019-\mathrm{nCoV}$ by using analysis of phylogenetic relationship for different species of bat coronaviruses including the novel 2019-nCoV. To recognize the relationship between bat coronaviruses and 2019-nCoV coronavirus, The analysis and comparison between the complete genome sequences of Spike (S) and Envelope (E) proteins of 20 available bat coronaviruses of different types including the novel 2019-nCoV were throughout the analysis of phylogenetics (Figure 1and Figure 2).

Spike protein phylogenetic tree analysis (Figures 1) illustrated that the 2019-nCoV in human can be seen gathered with many other different bat-coronaviruses. As it is observed in cluster $\mathrm{A}$ in (Figure $1 \mathrm{~A}$ ) the whole different bat-coronaviruses are observed clustered together which have very close relationship with 2019-nCoV. Different species of bat which are carrier for different coronavirus strains are Rousettus bat, bat-HKU9-10 strain, bat-SL-CoVZXC21, BtRs-BetaCoV/YN2018B, Rhinolophus bat (Figure $1 \mathrm{~B}$ ); therefore, It is clear that all the $2019-\mathrm{nCoV}$ related coronaviruses isolated from these species of bat are discovered in the mainland of China and Hong Kong. There are fruit bats (Rousettus bat) in Africa and Middle East but none of them are clustered in group A; Therefore, the Middle East bat are less likely to contribute in spreading or to become the origin of $2019-\mathrm{nCoV}[18,19,20,21]$. Furthermore, bat coronavirus BtCoV/279, Bat-SARS-coronavirus HKU3, and Bat-SARS-like-coronavirus isolate Rs4231 are observed to each other in one clade. All bat-coronaviruses of this group come from bats of Hong Kong and China isolated in different regions of China are observed in one clade next to the clade that contains $2019-\mathrm{nCoV}$ coronaviruses. Therefore, this group collected all coronaviruses that genetically are different from 2019-nCoV. [22]. Bat coronavirus come from bat of Hong Kong and USA but it does not contain the bats from China (Figure 1 C). Coronaviruses of this group are located into one clade far from the clade that contains $2019-\mathrm{nCoV}$ coronaviruses. Therefore all collected coronaviruses are genetically very different from 2019-nCoV and USA bat coronavirus may not has a role in generating of the2019-nCoV.

Spike protein phylogenetic analysis (Figures 1) illustrated all bat coronaviruses that clustered in group A are genetically far from the group that gathered in the clade that containing the 2019-nCoV (Figure 2 A). For group B, seven different bat-coronaviruses in China and USA are observed and it is composed of two sub clades. One clades contains more genetically relates coronaviruses but all are isolated from China and Hong Kong, and the second sub clade contains coronaviruses of bat that found in USA. It shows that the Envelope protein of different coronaviruses are more stable and more similar in comparison to Spike protein. In addition, USA coronavirus isolated from bat has relatively closeness related to the $2019-\mathrm{nCoV}$ but it is located in different sub clade and its spike sequence is very different and very far from to be concluded that USA bat coronavirus is related to the spreading of the $2019-\mathrm{nCoV}$ $[18,19,20,21]$. The multiple sequence alignments analysis of the full length amino acids for Envelope between different bat coronaviruses and including the 2019-nCoV. The amino acid alignments showed that all bat coronaviruses (NC 045512.2, MG772934.1, KY417146.1, 
MK211376.1, DQ648857.1, and DQ022305.2) that clustered in group B (Figure 2) have a similarity in amino acid sequence except that two amino acid have mutated (Figure 3) [23, 24]. This closely related amino acid sequence confirms that the results of phylogenetic tree analysis in which that these bat coronaviruses have genetically a close relationship together. Interestingly, one of these amino acid sequences, (MG772934.1) is 100\% identical with the amino acid sequence of the 2019n-CoV (NC 045512.2). The same virus has a close relationship in both Spike and Envelope amino acid sequence because in both phylogenetic tree analysis (Figure 1 and 2) are neighbored with the 2019n-CoV (NC 045512.2) in the same clade [25,26]. Therefore, this bat coronavirus, Bat-SARS-like-coronavirus isolate (bat-SLCoVZXC21) has a greatest probability to be the origin of the 2019-nCoV which are found in China but this type of bat is not exist in Iraq. The current outcomes have the comparability with many other international investigations findings $[18,21,23,24]$.

\section{CONCLUSION}

The findings of this investigation alongside with other international research analysis, it clearly reveal that all the 2019-nCoV related coronaviruses isolated from Rousettus bat, batHKU9-10 strain, bat-SL-CoVZXC21, BtRs-BetaCoV/YN2018B, Rhinolophus bat are discovered in the mainland of China and Hong Kong and there are Rousettus bat in the Middle East is less likely to contribute in spreading or to become the origin of 2019-nCoV. Bat coronavirus BtCoV/279, Bat-SARS-coronavirus HKU3, and Bat-SARS-like-coronavirus isolate Rs4231are genetically very different from the novel 2019-nCoV and USA coronavirus bat may has no role in generating of the 2019-nCoV. Moreover, the Envelope protein of different coronaviruses are more similar in comparison to Spike protein. In addition, USA coronavirus isolated from bat has relatively closeness related to the $2019-\mathrm{nCoV}$ but it is located in different sub clade and its spike sequence is very different and very far from to be concluded that USA bat coronavirus is related to the spreading of the $2019-\mathrm{nCoV}$. The outcome of this study aids in prediction and understand a possible origin of this new virus and importantly in the policy of infection control Also, development of a subunit vaccine in improving the human immunity against COIVD-19. Therefore, there is a requirement to identify the novel 2019-nCoV close relationship with other animal coronaviruses, the alignments of structural proteins of the 2019-nCoV and finally analysis of phylogenetics for the whole viral genome obtained in each geographical places.

\section{ACKNOWLEDGEMENT}

We acknowledge the presidency of Sulaimani Polytechnic University and especially Assistant Professor Dr. Alan Faraydoon Ali for his great help and academic support for performing this research. Also, we would like to appreciate Mam Humanitarian Foundation for their financial and logistic support.

\section{REFERENCE}

[1] C. Drosten, S. Günther, W. Preiser, et al. Identification of a novel coronavirus associated with severe acute respiratory syndrome. N Engl J Med.; 348:1967-1976, 2003.

[2] Y. Chen, Q. Liu, D. Guo. Emerging coronaviruses: genome structure, replication, and pathogenesis. J Med Virol., 2020. https://doi.org/10. 1002/jmv.25681

[3] JF. Chan, S. Yuan, KH. Kok, et al. A familial cluster of pneumonia associated with the 2019 novel coronavirus indicating person-to- person transmission: a study of a family cluster. Lancet., 2020. https:// doi.org/10.1016/S0140-6736(20)30154-9

[4] C. Huang, Y. Wang, X. Li, et al. Clinical features of patients infected with 2019 novel coronavirus in Wuhan, China. Lancet., 2020. https://doi. org/10.1016/S0140-6736(20)30183-5

[5] N. Zhu, D. Zhang, W. Wang, et al. A novel coronavirus from patients with pneumonia in China, 2019. N Engl J Med., 2020. https://doi.org/ 10.1056/NEJMoa2001017

Coronavirus disease 2019 (COVID-19) Situation Report - 74, World Health Organization (WHO). https:/www.who.int/docs/default-source/coronaviruse/situation-reports/20200403-sitrep-74-covid-19mp.pdf?sfvrsn=4e043d03 12

[7] H. Lu, CW. Stratton, YW. Tang. Outbreak of pneumonia of unknown etiology in Wuhan China: the mystery and the miracle. J Med Virol., 2020. https://doi.org/10.1002/jmv.25678

[8] W. Ji, W. Wang, X. Zhao, J. Zai, X. Li. Homologous recombination within the spike glycoprotein of the newly identified coronavirus may boost cross-species transmission from snake to human. J Med Virol., 2020. https://doi.org/10.1002/jmv.25682 
[9] DC. Scales, K. Green, AK. Chan, SM. Poutanen, D. Foster, K. Nowak, et al. Illness in intensive-care staff after brief exposure to severe acute respiratory syndrome. Emerging Infect Dis.; 9:1205-1210, 2003. https://oi.org/10.3201/eid0910.030525

[10] K. Mizumoto, G. Chowell, Estimating Risk for Death from 2019 Novel Coronavirus Disease, China, JanuaryFebruary 2020. Retrieved from Volume 26, Number 6-June 2020. Center for Disease Control and Prevention -CDC. https://doi.org/10.3201/eid2606.200233

[11] Y. Chen, Q. Liu, D. Guo, Emerging coronaviruses: Genome structure, replication, and pathogenesis. J. Med. Virol., 2020. https://doi.org/10.1002/jmv.25681

[12] J. Cai, W. Sun, J. Huang, M. Gamber, J. Wu, G. He, Early Release - Indirect Virus Transmission in Cluster of COVID-19 Cases, Wenzhou, China. Emerging Infect. Dis. 26(6), 2020 https://doi.org/10.3201/eid2606.200412

[13] K. Marie, B. Holly, M. Claire, G. Susan, W. John. Middle East Respiratory Syndrome Coronavirus Transmission. Emerging Infect. Dis. www.cdc.gov/eid • Vol. 26, No. 2, 2020. https://doi.org/10.3201/eid2602.190697

[14] P. Forster, L. Forster, C. Renfrew, M. Forster. Phylogenetic network analysis of SARS-CoV-2 genomes. PNAS, www.pnas.org/cgi/doi/10.1073/pnas.2004999117,2020.

[15] TA. Hall. BioEditA user-friendly biological sequence alignment editor and analysis program for Windows 95/98/NT. Nucleic Acids Symp Ser.; 41:95-98, 1999. https://doi.org/10.14601/Phytopathol_Mediterr14998 u 1.29

[16] S. Kumar, G. Stecher, M. Li, C. Knyaz, K. Tamura. MEGA X: Molecular Evolutionary Genetics Analysis across computing platforms. Mol. Biol. Evol. 2018;35(6):1547-1549. https://doi.org/10.1093/molbev/msy096

[17] A. Dereeper, et al., Phylogeny.fr: robust phylogenetic analysis for the non-specialist. Nucleic Acids Res. 2008 Jul 1; 36 (Web Server issue): W465-9.

[18] P. Zhou, et al. A pneumonia outbreak associated with a new coronavirus of probable bat origin. Nature, 2020. https://doi.org/10.1038/s41586-020-2012-7.

[19] D. Hu, C. Zhu, L. Ai L, et al. Genomic characterization and infectivity of a novel SARS-like coronavirus in Chinese bats. Emerg Microbes Infect 2018; 7: 154.

[20] S. Lau, K. Woo, Y. Li, H. Huang, B. Tosi, S. Wong, S. Wong, K. Leung, H. Chan, K. Yuen. Severe acute respiratory syndrome coronavirus-like virus in Chinese horseshoe bats. Proc. Natl. Acad. Sci. USA 102:14040 14045,2005

[21] B. Hu, L. Zeng, X. Yang, et al. Discovery of a rich gene pool of bat SARS-related coronaviruses provides new insights into the origin of SARS coronavirus. PLoS Pathog, 13, Article e1006698, 2017.

[22] T. Zhang, Q. Wu, Z. Zhang. Pangolin homology associated with2019-nCoV. BioRxiv, 2020. https://doi.org/10.1101/2020.02.19.950253.

[23] C. Huang, W. Liu W. Xu, T. Jin, Y. Zhao, J. Song, Y. Shi, W. Ji, H. Jia, Y. Zhou, et al. A bat-derived putative cross-family recombinant coronavirus with a Reovirus gene. PLoS Pathog, 12, p. e1005883, 2016. https://doi.org/10.1371/journal.ppat.1005883.

[24] K. Andersen, A. Rambaut, W. Lipkin, E. Holmes, R. Garry, The Proximal Origin of SARS-CoV-2. ARTIC Network, 2020. https://doi.org/10.1038/s41591-020-0820-9

[25] P. Woo, S. Lau, C. Lam, A. Tsang, S. Hui, R. Fan, P. Martelli, K. Yuen. Discovery of a novel bottlenose dolphin coronavirus reveals a distinct species of marine mammal coronavirus in Gammacoro- navirus. J Virol 88:1318 -1331, 2014. http://dx.doi.org/10.1128/JVI.02351-13.

[26] S. Ali, T. Hama-Soor, M. Babakir-Mina, S. Dimonte, F. Greco. Is It Scaly Anteater or Bat A Real Origin of The 2019-Novel CoV: A Probable Hypothesis?. Kurdisatn Journal of Applied Research (KJAR), Volume 5 (1): 1 12. http://dx.doi.org/10.24017/covid.1

Kurdistan Journal of Applied Research $\mid 4^{\text {th }}$ International Conference on the Health and Medical Science: Medical Researches Improve Life Quality (ICHMS 2020) | 144 